\title{
Categorizing mathematical sense making and an example of how physics understanding can support mathematical understanding
}

\author{
Julian D. Gifford and Noah D. Finkelstein \\ Department of Physics, University of Colorado Boulder, Box 390, 80309
}

\begin{abstract}
For both instructors and researchers there is interest in student "sense making" and specifically on mathematical sense making. Here, we introduce a framework that distinguishes between the object of the sense making (mathematical or physical) and the tool used for sense making (formal mathematics or conceptual physics), thus foregrounding focus and mechanism in student problem solving. We situate this framework in both a general discussion of sense making and of mathematical sense making in particular, and then apply it to data from a focus group. This analysis highlights a particular mode of sense making, where a conceptual understanding of a physical system is used as a tool to understand a mathematical object. While we begin to demonstrate its utility in describing the individual moves that students make, as well as the coordination and sequencing of these moves, when engaged in a larger mathematical sense making activity, we anticipate that the framework will also provide a guide for the design and development of curricula that support these sense making moves.
\end{abstract}

2019 PERC Proceedings edited by Cao, Wolf, and Bennett; Peer-reviewed, doi.org/10.1119/perc.2019.pr.Gifford Published by the American Association of Physics Teachers under a Creative Commons Attribution 4.0 license. Further distribution must maintain attribution to the article's authors, cover page, and DOI. 


\section{INTRODUCTION}

In the PER and science education communities, much attention has been given to the construct of "sense making." This work has been reviewed and synthesized by Odden and Russ, leading to a general definition of sense making as "the process of building an explanation to resolve a perceived gap or conflict in knowledge" [1]. In addition to this broad definition, Odden and Russ distinguish three "strands" of sense making in the science education literature in which sense making is seen as (1) an epistemological stance towards science learning (2) a cognitive process and (3) a specific discourse practice. The first strand requires that, for a given reasoning activity to count as sense making, the individual (or group) must hold a belief that the ultimate purpose of the activity is the construction of an explanation leading to a greater understanding of a given phenomenon - not simply the creation of an answer. The second strand highlights sense making as a cognitive activity that leads to the construction of new knowledge or novel connections between existing knowledge elements. The final strand distinguishes sense making from "thinking" or "argumentation" more generally, focusing again on the construction of an explanation for the purpose of understanding, not persuading.

Situating our work in this general space of sense making, we consider the more specific topic of mathematical sense making (MSM). Recent work on MSM has used varied definitions of sense making; considering it to be the coordination of conceptual understanding with the use and interpretation of algebraic symbols [2] or "coherence seeking" between mathematical formalisms and functional relationships in the world [3]. We see both of these definitions as steps towards specifying the cognitive process strand in the Odden/Russ definition of sense making. In considering mathematical problem solving more generally, there exist both student-centric problem solving strategies aimed at helping students engage with mathematical formalisms more like experts [4-6] and researcher-centric frameworks for considering student use of mathematics in physics [7-10]. However, many of these frameworks highlight either the mechanism or the object of the sense making, but tend not to foreground both. As part of a larger project aimed at understanding student sense making and the design of curricula to support this sense making, we have developed a framework that builds on these existing models, but attends to both the mechanism and the object of sense making. Distinguishing between these, while simultaneously attending to both, allows for a more nuanced understanding of student reasoning and (though we do not discuss this presently) provides greater specificity in considering the actions we wish students to engage in when designing curricula. This framework highlights the cognitive process and discourse practice strands to look for specific ways in which sense making might occur when considering the use of formal mathematics in physics.

\section{THE FRAMEWORK}

While Odden and Russ provide a definition for sense making in general, we consider this framework in the context of mathematics and physics. Vygotsky introduced the idea of mediated cognition as the basis for all higher order cognitive processes; here a mediator, or "tool," is employed to aid in a subject's understanding of an object, as a complement to the individual's direct interaction with that object [11]. A common schematic of this cognitive structure is a triangle with the vertices subject, object and tool. The line connecting the subject and the object represents a direct engagement with (or understanding of) the object (e.g., developed through physical experimentation and embodied cognition [12]) and the legs connecting the subject and object through a tool represent a mediated understanding of the object scaffolded by the use of the particular tool (e.g. using language - or mathematics - to describe and indirectly interact with the object).

Applying the notion of mediated cognition leads us to consider instances of physical sense making where mathematics serves as either (1) the tool and/or (2) the object of the cognitive process. This framing allows us to begin to operationalize the first strand of Odden and Russ's sense making (figuring something out) in that we begin by categorizing what it is that is being figured out - the object of the sense making while simultaneously highlighting the second strand (cognitive process) by considering how it is being figured out - what tool is being used and how is it employed. Like Sayre [10] we distinguish between physics and mathematics, we then specify four reasoning structures, or sense making modes, in the context of physics: mathematical sense making of a mathematical object (Msm-M), mathematical sense making of a physical object (Msm-P), physical sense making of a mathematical object (Psm-M), and physical sense making of a physical object (Psm-P). The four modes are shown in Fig.1 and are briefly explained below.

Psm-P: Much of the early work in PER focused on determining and cataloging common student difficulties with the concepts of introductory physics. This basic research was then used as a base for the development of curricula. These research-based curricula generally aimed to develop conceptual reasoning skills to help students build physical models (often excluding formal mathematics) to describe physical phenomenon. A canonical example comes from the University of Washington's study of student reasoning regarding electric circuits $[13,14]$. The curricula developed out of these studies [15] scaffold students in building a conceptual, qualitative model of electric circuits, encouraging the use of physical reasoning as a tool for understanding a physical system. Using the framework shown in Fig.1, we consider these curricula to engage students in a Psm-P mode of reasoning. While we do not consider this mode in isolation to be an example of mathematical sense making, the coordination of Psm-P with the other modes can contribute to a larger activity of MSM - as shown in Part III below. This example highlights a major affordance of this framework: having op- 


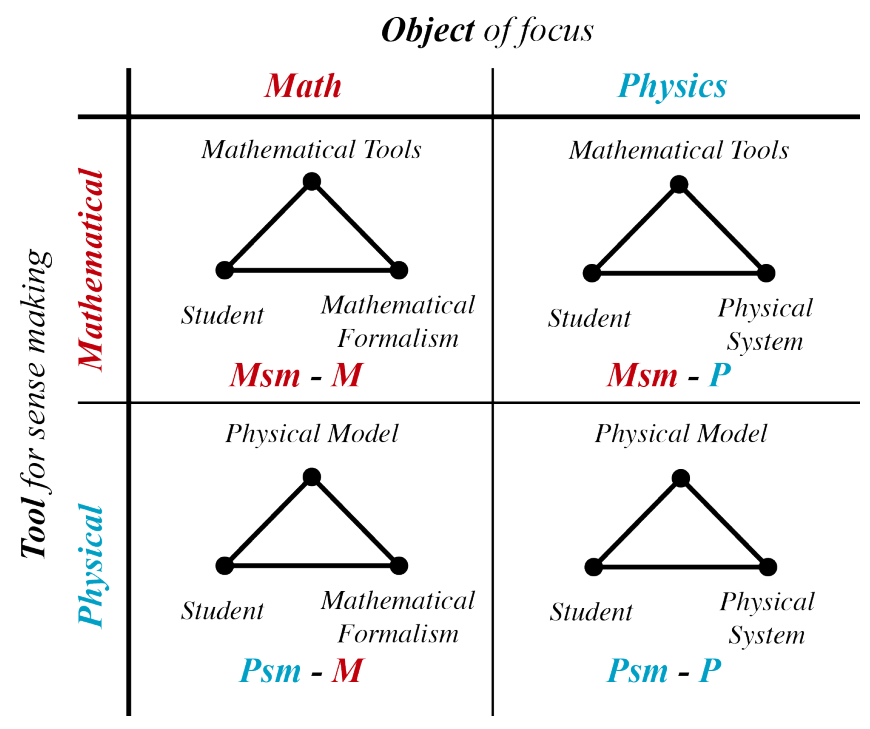

FIG. 1. The four reasoning structures (modes) of our sense making framework. These modes differ based on the object of the sense making and the tool employed in the sense making.

erationalized these sense making modes, we can analyze not only student reasoning but also curricula by considering the reasoning structures (and the connections between them) that it scaffolds students to use.

Msm-M: Perhaps the most "mathematical" mode would be using mathematical reasoning as a tool for understanding a mathematical object (Msm-M). In this mode mathematical reasoning is utilized to understand an object that is mathematical in nature (an equation, graph, etc.) and does not focus on any connection to the physical system. This is not to suggest that the mathematical object does not have physical significance, rather that in an Msm-M mode these physical properties are not the focus of attention. Work by Hahn et. al [2] studied physics sense making strategies focusing largely on answer-checking types of reasoning. In class and on homework, after obtaining a mathematical answer, students were explicitly asked to consider what kind of "beast" (mathematical entity) it was as well as the overall functional behavior. While students were then asked to assess the appropriateness of their responses for the given physical scenario (requiring students to engage in another mode, e.g. Psm-P, and compare their physical reasoning to their mathematical expression), the first moves of these particular sense making strategies ignore this physical connection and treat the expression as a purely mathematical object. As such, we view these moves as examples of Msm-M. The larger activity of physics sense making studied by Hahn involves the coordination of multiple reasoning structures (i.e. Msm-M and Psm-P); while we view this coordination of multiple modes as an integral component of sense making, we also find utility in categorizing the individual reasoning structures involved.

Msm-P: Perhaps the most canonical view of MSM would be instances when a mathematical formalism is leveraged to understand the behavior of a physical system. In practice, this is often a complicated process, perhaps requiring Msm$M$ type reasoning to understand the formalism before it can be used to understand physical behavior. Despite this complicated structure, it is still possible to identify local instances of Msm-P, such as in Kuo's analysis of a mathematical "shortcut" in which a student leverages their understanding of the expression $v_{f}=v_{0}+a t$ as describing a "base plus change" process to determine the difference between the final velocities of two balls thrown with different initial velocities [16]. In their study, Kuo et. al found that several students explicitly mentioned the expression above and then used the conceptual argument that "since the initial difference in velocities is 2 meters per second, and since both balls undergo the same change in velocity, the final difference in velocities is still 2 meters per second". This example shows that a larger sense making activity can be seen as involving multiple reasoning modes, in this case the use of Msm-M reasoning (interpreting the equation as base plus change) to subsequently engage in Msm-P (using their interpretation of the equation as a scaffold to consider the changes in velocity of the balls).

Psm-M: In the final mode of our framework, physical reasoning is used as a way to understand a mathematical object. As with Msm-P, this mixed reasoning mode is likely done in conjunction with another mode (e.g. engaging in Psm-P in order to leverage a conceptual understanding of the system to understand the mathematical formalism). However, we still observe local instances of Psm-M, for example OSU's use of three-dimensional surfaces as a way to understand and conceptualize partial derivatives [17].

We do not believe that there is a hierarchy to these modes, i.e. we do not see one of these as better or more important than the others, rather we see each as a potentially productive reasoning structure when considering sense making of physical and mathematical phenomenon. Part of the utility of this framework is that it provides a language for discussing the small-scale actions that students engage in. However, we are not attempting to redefine sense making or claim that these modes are a complete description of all possible sense making, rather we are attempting to operationalize the moves students engage in that might contribute to a broader activity of MSM. We view these moves essentially as "atoms" of reasoning, and in our analysis look for the ways in which these modes are assembled and chained together into "molecules" of sense making.

While we believe this framing provides a productive way of considering student reasoning in general, we find it to be particularly useful in describing MSM in a way that is situated in the sense making literature and inclusive of the many existing frameworks for student use of mathematics. In addition to its focus on the first two strands of Odden and Russ's definition, we believe this framework also addresses the third strand by providing a language for categorizing modes of reasoning (and so types of arguments) that students may rely on as they discuss and argue towards an explanation of mathematical and physical phenomena. While future work will 
discuss the affordances of this framework in greater depth, in the analysis that follows we consider an episode of student reasoning that highlights primarily the Psm-M mode, as well as the ability to distinguish between multiple modes in a single reasoning episode. We chose to present data that showcases this mode to demonstrate the utility of this framework in describing a reasoning modality that is less commonly discussed in physics, i.e. how physical reasoning supports understanding of mathematics, and to show that students can engage in and shift between these modes rapidly. The analysis was agreed upon by both authors and was discussed in detail in the professional setting of a research group. It is used to show face-validity of the framework, we make no claims regarding the ubiquity of student reasoning here.

\section{APPLICATION OF THE FRAMEWORK}

The data presented here come from a one-time focus group conducted at the end of the semester of a modern physics course primarily for engineering majors (largely mechanical and electrical). The participants are three students from this course: Amanda ("A", female), Ben ("B", male), and Jack ("J", male). The second author is the professor of record and the first author played a substantial role in both curricular development and in-class implementation. A major focus of the course is the development of a holistic model for light, which includes an in depth consideration of the photoelectric effect and the photon model. At multiple points throughout the course - a pretest (week 1), a homework (week 3), and a free response exam question (week 5) - these students were asked to consider a plot of the maximum kinetic energy of the ejected electrons versus the wavelength of incident light (in addition to the more canonical plot of KE $\mathrm{v}$ frequency) in a photoelectric experiment. While a plot of KE versus frequency is linear, KE is inversely proportional to wavelength. Further evidence for the photon model comes from the cutoff frequency, the minimum frequency of light that will eject electrons from the plate; due to the inverse relationship between frequency and wavelength, this minimum frequency becomes a maximum wavelength above which the $\mathrm{KE}$ is zero as no electrons are ejected. Qualitatively accurate plots of $\mathrm{KE} \vee f$ and $\mathrm{KE} \mathrm{v} \lambda$ are shown in the left and center graphs of Fig. 2, while student work is shown in the rightmost graph. In the data below, these students were asked to draw plots of $\mathrm{KE} \mathrm{v} \lambda$ for plates of two different work functions (a measure of how tightly bound the electrons are, and so the minimum photon energy required to eject electrons from the plate). In the transcripts that follow, ellipses represent omissions in the complete student dialogue made for clarity and length.

A: I was gonna draw something sorta like, uhhh, that [crossed out plots in rightmost graph of Fig. 2]. 'Cause uhh the shorter the wavelength is the more powerful the light is so the more energy this [the electron] has when it bounces, when it comes off of the plate.

J: And then the other one with the different uhh work func- tion would be always less than that? Or, because wouldn't it [the electron] always have less kinetic energy because it leaves with less?

A: Well, I think it would still have the same like asymptotes here $[\lambda=0] \ldots$ but like it would dip further down closer to this [the $\lambda$ axis].

J: And remain under it [the first plot] the whole time.

In this exchange Amanda and Jack are attempting to generate a graph of KE vs. $\lambda$. While this graph inarguably has physical significance, in this moment we see this plot as a fundamentally mathematical object, expressing a relationship between two variables. However, Amanda's reasoning is physical in nature (that shorter wavelength light is more powerful, and so the electrons will leave with more energy). We take this as evidence that Amanda is engaged in a Psm-M mode of reasoning. Jack also joins in this mode, suggesting that the second plot be "always less than" the first because electrons are ejected from the second plate with less energy (for the same wavelength). While one might argue that Amanda's focus on the inverse relationship between wavelength and energy is mathematical in nature, her use of the phrase "more powerful" suggests to us that she is reasoning about relationships between physical quantities, rather than mathematical variables. At this point, the students have agreed that the plots should be inverse decays (they have drawn the incorrect crossed out plots in Fig.2) but have not indicated the cutoff wavelengths or their relation to the work function and have not used any explicit mathematical formalisms to scaffold their reasoning. The students feel they have answered the question fully (Amanda saying "well that's this round of questions") at which point the interviewer (the first author) asks them if there are any relevant mathematical equations that might model the graphs they have drawn. The students agree that there is, but that they don't remember it. After a period of "equation hunting" through their notes, Ben suggests:

B: These [the plots] are definitely inverse relationships. What about, possibly, it seems to follow the relationship of KEmax over $\lambda$. As $\lambda$ increases this [the graph] decreases like a curve.

A: ...You're right about the $\lambda$ but... 'cause we're graphing, the input is $\lambda$ the output is the kinetic energy max, so we'd have $K E=\frac{1}{\lambda}$, well some expression over $\lambda$ because it varies how much of sorta a dip that that has. But the basic one would be something over $\lambda$.

The object here is mathematical (a symbolic expression) and both Ben and Amanda's reasoning is primarily mathematical in nature (Ben arguing that it should be a $\frac{1}{\lambda}$ relationship because of the shape of the graph, and Amanda treating $\mathrm{KE}$ and $\lambda$ not as physically relevant constructs but as mathematical variables (inputs and outputs). Thus, we classify this exchange as an example of Msm-M, where the plot they've drawn scaffolds the creation of a symbolic formalism. This is a shift in reasoning structure from earlier, where they were largely relying on physical reasoning. Following this exchange, the students attempt to determine what the "something" over $\lambda$ should be, eventually coming to the (correct) 

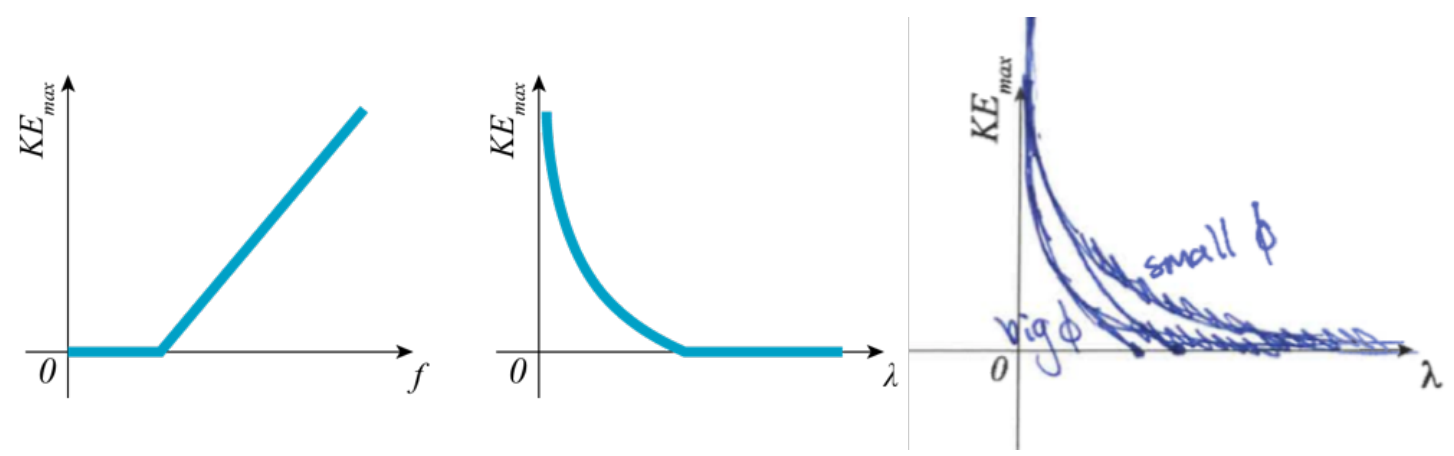

FIG. 2. Left and center graphs show accurate plots of $\mathrm{KE} \mathrm{v} f$ and $\mathrm{KE} \mathrm{v} \lambda$ respectively. Rightmost graph shows student work from Amanda. Initially she drew two inverse decays that decay at different rates (the crossed-out plots in this figure). Eventually she goes back to correct this to show two identical decays with different shifts, hitting the horizontal axis at different values of $\lambda$.

expression $K E=\frac{h c}{\lambda}-\Phi$. In considering the validity of this expression, Amanda remains in an Msm-M mode, relating pieces of the equation to their effect on the plot: "...that $\left[\frac{h c}{\lambda}\right]$ would give us the shape of this graph and then that $[-\Phi]$ would give us our shift based on the work function." The interviewer then asks how $\Phi$ shifts the graph, which leads the group to shift between three modes as they address a contradiction between the formal mathematics and their understanding of the photoelectric effect. To highlight the rapid shifts in reasoning, we have explicitly noted the coded modes in the transcript below.

Msm-M:

A: Normally in a shift graphically it shifts your asymptotes too... But in our graph we decided that it wasn't going to shift the asymptotes... But then if this [their equation] is right then it would mean that the horizontal asymptote would shift down as well.

Psm-P:

B: But wait, if the work function is stronger then the electrons wouldn't have any kinetic energy.

A: That's true it would eventually go to zero, it would make the kinetic energy negative but

B: I don't think that's possible yet, gotta wait till tunneling ...

A: Well, eventually, eventually ... no electrons will be coming off the plate once $\lambda$ gets long enough. It won't have enough energy.

Psm-M:

J: Oh yeah, so it'll just drop off... they'll [the graphs] just end.

A: Mhmm. Which would be based on that work function... Once $\lambda$ gets big enough and it has no kinetic energy because nothing is being ejected... And then the larger work function would still be up here [asymptote at infinity for $\lambda=0$ ] and it would kinda hit like that [smaller $\lambda$ intercept]... And then this equation could describe it because it would be shifting that graph down but $\mathrm{KE}$ always has to be positive so the graph would just end where it intercepts that axis.

B: Yeah, sounds about right. It's more scientifically accurate. In this exchange Amanda notes a conflict between their mathematical expression and their knowledge of photoelectric phenomenon. By establishing that kinetic energy cannot be negative, instead meaning no ejection and so zero KE, they use physical reasoning to alter their initial graphs. They apply this same physical reasoning to their symbolic expression and determine that the expression only works "when $\frac{h c}{\lambda}$ is greater than or equal to $\Phi$... Otherwise you might have a negative kinetic energy at a large enough wavelength." We view this as an example of Psm-M, where physical reasoning is employed as a tool not only to understand an existing mathematical object, but also to generate mathematical objects and determine when they are valid. Further evidence for their engagement in Psm-M comes from Ben's statement that their modified plots and equation are more "scientifically accurate", suggesting that there is a strong relationship between the mathematical objects and the physical phenomena they describe. While the entire reasoning episode might be classified as an example of Psm-M, at a finer grained analysis we see this "molecule" of sense making includes the coordination of three different reasoning modes.

\section{CONCLUSIONS}

We have introduced a framework for MSM that emphasizes both the mechanism and object of sense making, connecting this to both prior work in student use of mathematics and also to a broad definition of sense making approaches. Through a case study that highlights one mode of reasoning (Psm-M), we have shown the utility of this framework in analyzing student reasoning and the attention it brings to the shifts and connections between modes. Future work will examine all four modes in greater detail, and explore the sequencing and coordination of these modes. We hope to employ this framework as a tool for both understanding student reasoning and the development and design of curricula.

Acknowledgements: The authors would like to thank the $\mathrm{CU}$ and Maryland PER groups for helpful feedback and discussion. This work was supported by NSF: 1625824. 
[1] T. O. B. Odden and R. S. Russ, Defining sensemaking: Bringing clarity to a fragmented theoretical construct, Science Education, 1-19 (2018)

[2] K. T. Hahn, Student Evaluative Sensemaking on Homework in Intermediate Mechanics, Masters Thesis, Oregon State University (2018).

[3] B. W. Dreyfus et. al, Mathematical sense-making in quantum mechanics: An initial peek, Phys Rev. ST Phys. Educ. Res. 13 020141 (2017).

[4] J. I. Heller \& F. Reif, Prescribing Effective Human ProblemSolving Processes: Problem Description in Physics, Cognition and Instruction 1 (1984).

[5] A. Van Heuvelen, Learning to think like a physicist: A review of research-based instructional strategies, Am. J. Phys. 59, 891 (1991).

[6] L. Hsu, E. Brewe, T. M. Foster, \& K. A. Harper, Resource letter RPS-1: Research in problem solving, Am. J. Phys, 72(9), (2004).

[7] B. R. Wilcox, et al., Analytic framework for students' use of mathematics in upper-division physics, Phys. Rev. ST Phys. Educ. Res. 9, 020119 (2013).

[8] T. J. Bing and E. F. Redish, Analyzing problem solving using math in physics: Epistemological framing via warrants, Phys. Rev. ST Phys. Educ. Res. 5, 020108 (2009).

[9] O. Uhden, et al., Modelling Mathematical Reasoning in
Physics Education, Sci. \& Educ. 21(4), (2012).

[10] B. Modir, J. D. Thompson, \& E. C. Sayre, Students' epistemological framing in quantum mechanics problem solving, Phys. Rev. ST Phys. Educ. Res. 13, 020108 (2017).

[11] L. Vygotsky, Mind in Society (Harvard University Press, 1978).

[12] G. Lakoff and R. Nunez Where Mathematics Comes From (Basic Books, 2000).

[13] L. C. McDermott and P. S. Shaffer, Research as a guide for curriculum development: An example from introductory electricity. Part I: Investigation of student understanding, Am. J. Phys 60 (1992).

[14] P. S. Shaffer and L. C. McDermott, Research as a guide for curriculum development: An example from introductory electricity. Part II: Design of instructional strategies, Am. J. Phys 60 (1992).

[15] L. C. McDermott and the Physics Education Group at the University of Washington, Physics by Inquiry Vols. 1 and II (New York: John Wiley \& Sons, Inc., 1996) and L. C. McDermott, P. S. Shaffer, and the Physics Education Group at the University of Washington Tutorials in Introductory Physics (1991Present).

[16] E. Kuo et al., How students blend formal and conceptual mathematical reasoning in solving physics problems, Science Education, 97: 32-57 (2013).

[17] https://osuper.science.oregonstate.edu/content/ raising-physics-surface. 